\title{
Medical Quiz : SBA
}

\section{Questions (www.medicinepd.co.uk)}

1. Following a routine blood and biochemical screen a 55-year-old woman was found to have isolated hypercalcaemia of 3.10mmol/l (2.1-2.5). She had no symptoms. Further investigation showed raised parathormone (PTH) but no evidence of a multiple endocrine neoplasia syndrome (MEN1 or MEN2). A diagnosis of primary hyperparathyroidism (PHPT) was made. Regarding her further management the indication for SURGICAL treatment would be:
a. a 24 hour urinary calcium excretion $>400 \mathrm{mg}$
b the risk of an underlying parathyroid carcinoma
c. the high risk of bone fracture if managed conservatively

d. her age, making it very likely that the disease will progress over the coming years

e. the low risks of surgery failing to correct the condition or cause permanent hypoparathyroidism

\section{Regarding infective endocarditis (IE) it is TRUE that:}

a. a peripheral clinical manifestation (Janeway lesion, Osler node or splinter haemorrhage) is found in the majority of cases

b. three sets of blood cultures from different sites are recommended to be taken in the first 2 hours before initiating treatment in suspected cases

c. streptococci are currently the leading cause

d. after removal of an infected pacing system antibiotic treatment of a known sensitive organism can be discontinued after 4 weeks

e. a 2-week course of once daily treatment of a sensitive streptococcus in the absence of significant valvular regurgitation, emboli or heart failure, can be undertaken in the home

3. The following complication has NOT been described in Campylobacter jejuni gastrointestinal infections:
a. Arthritis
b. Guillain-Barré syndrome(GBS)
c. Haemolytic uraemic syndrome (HUS)
d. Lymphoma of the small intestine
e. Osteomyelitis 